\title{
Solar proton events in cosmogenic isotope data
}

\author{
Ilya G. Usoskin, ${ }^{1}$ Sami K. Solanki, ${ }^{2}$ Gennady A. Kovaltsov, ${ }^{3}$ Jürg Beer, ${ }^{4}$ \\ and Bernd Kromer ${ }^{5}$ \\ Received 16 February 2006; revised 22 March 2006; accepted 30 March 2006; published 27 April 2006.
}

[1] A possible contribution of solar energetic particle events to the production of cosmogenic ${ }^{10} \mathrm{Be}$ and ${ }^{14} \mathrm{C}$ in the atmosphere is studied. The solar particle effect is negligible in the ${ }^{14} \mathrm{C}$ data, but extreme events may be detectable in high-resolution ${ }^{14} \mathrm{C}$ data. Although the overall effect is small in the ${ }^{10} \mathrm{Be}$ data, strong events may contribute notably on the inter-annual time scale. In combination with the 11-year solar modulation of galactic cosmic rays, it may lead to an intermittent 5.5-year periodicity, which is seen in high resolution ${ }^{10} \mathrm{Be}$ data. We have identified ten episodes during $1750-1950$ when ${ }^{10} \mathrm{Be}$ may hold signatures of strong solar proton events. This opens a new possibility to study extreme solar particle events in the past using high resolution cosmogenic isotope data. Citation: Usoskin, I. G., S. K. Solanki, G. A. Kovaltsov, J. Beer, and B. Kromer (2006), Solar proton events in cosmogenic isotope data, Geophys. Res. Lett., 33, L08107, doi:10.1029/2006GL026059.

\section{Introduction}

[2] The content of cosmogenic isotopes in terrestrial archives $\left({ }^{14} \mathrm{C}\right.$ in tree rings and ${ }^{10} \mathrm{Be}$ in polar ice) serves as a proxy of the cosmic ray intensity in the past. The bulk of cosmogenic isotopes is produced by energetic galactic cosmic rays (GCR), which are modulated by solar magnetic activity, thus providing a commonly accepted link between the cosmogenic isotopes and solar activity on the long-term scale. Recent reconstructions of solar activity in the past [McCracken et al., 2004; Solanki et al., 2004; Usoskin et al., 2003, 2004] are based on cosmogenic isotope data and physical models of the isotope production by GCR. However, during a sporadic solar proton event (SPE), the flux of lower energy particles near the Earth may increase by orders of magnitude. Can such SPE produce a significant amount of cosmogenic isotopes to affect the reconstructed past solar activity? A possible SPE effect in cosmogenic isotope data has been discussed in the past with controversial results. For example, theoretical calculations of SPE contribution into ${ }^{14} \mathrm{C}$ production [Lingenfelter and Ramaty, 1970; Castagnoli and Lal, 1980; Masarik and Reedy, 1995; Dorman, 2004] vary by two orders of magnitude. Experimental searches for SPE signatures in ${ }^{14} \mathrm{C}$ were also contradictory. For example, Damon et al. [1989] reported a notable SPE signal in $\Delta^{14} \mathrm{C}$ during 1930-1950, but a similar analysis by Stuiver and

\footnotetext{
${ }^{1}$ Sodankylä Geophysical Observatory, Oulu, Finland.

${ }^{2}$ Max-Planck-Institut für Sonnensystemforschung, Katlenburg-Lindau, Germany.

${ }^{3}$ Ioffe Physical-Technical Institute, St. Petersburg, Russia.

${ }^{4}$ EAWAG, Dübendorf, Switzerland.

${ }^{5}$ Heidelberger Akademie der Wissenschaften, Heidelberg, Germany.
}

Copyright 2006 by the American Geophysical Union. 0094-8276/06/2006GL026059\$05.00
Braziunas [1998] was consistent with GCR-dominated production. A careful study of ${ }^{14} \mathrm{C}$ data gives no evidence for a direct SPE signature [Bartolomei et al., 1995]. A small $\approx 10 \%$ relative increase was found in atmospheric ${ }^{14} \mathrm{CO}$ [Jöckel et al., 2003] in association with a strong SPE in 1989 , but it is too small to produce a notable signature in the tree-ring data. We are not aware of any detailed calculations of the SPE effect for ${ }^{10} \mathrm{Be}$ production in the Earth's atmosphere.

[3] Here we perform a thorough quantitative study of a possible SPE signal in terrestrial cosmogenic isotope data.

\section{Cosmogenic Isotope Production}

[4] GCR are always present in the vicinity of Earth, but their flux and energy spectrum are subject to modulation by solar magnetic activity (Figure 1). Here we consider the GCR spectrum parameterized by the force-field approximation [see, e.g., McCracken et al., 2004; Usoskin et al., 2005].

[5] The differential energy spectrum of solar particles is usually parameterized as an exponent in rigidity

$$
J(P)=J_{0} \cdot \exp \left(P / P_{0}\right)
$$

where $P_{0}$ is the characteristic rigidity of the spectrum [Freier and Webber, 1963]. Energy spectra of SPEs computed in this approximation are shown in Figure 1. Solar particles dominate over the GCR flux in the energy range below $100 \mathrm{MeV}$, but during years with intense SEP events this may shift to energies of up to $1 \mathrm{GeV}$. The strength of a SPE is usually characterized by the total omnidirectional fluence of particles with energy above $30 \mathrm{MeV}, F_{30}$. To be consistent with annual cosmogenic isotope data, here we consider the annual fluence. Annual values of $F_{30}$ and $P_{0}$ for the last 50 years were adopted from Shea and Smart [1990], Feynman et al. [1993] and IMP-8 data (http://sd-www.jhuapl.edu/IMP). Typical values for the last decades are: $P_{0}$ varies between $50 \mathrm{MV}$ and $300 \mathrm{MV}$ (the mean value $\left.\left\langle P_{0}\right\rangle=78 \mathrm{MV}\right) ; F_{30}$ reaches up to $10^{10} \mathrm{~cm}^{2}$ $\left(\left\langle F_{30}\right\rangle=10^{9} \mathrm{~cm}^{2}\right)$.

[6] Using the differential energy spectra and the specific yield functions of isotope production (by Castagnoli and Lal [1980] for ${ }^{14} \mathrm{C}$ and by Webber and Higbie [2003] for ${ }^{10} \mathrm{Be}$ ), one can calculate the expected cosmogenic isotope production rate $Q$. The processes of atmospheric transport and subsequent deposition are quite different for the two isotopes. ${ }^{14} \mathrm{C}$ becomes oxidized to $\mathrm{CO}_{2}$ and takes part in the global carbon cycle, which results in the global mixing of ${ }^{14} \mathrm{C}$ before its capture by living trees. The atmospheric transport of ${ }^{10} \mathrm{Be}$ is more straightforward but less clear. ${ }^{10} \mathrm{Be}$ produced in the troposphere can precipitate quickly, thus being deposited locally. Stratospheric ${ }^{10} \mathrm{Be}$ resides in 


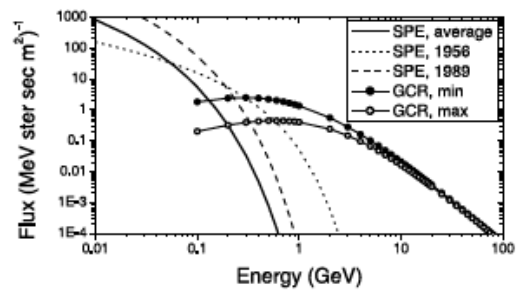

Figure 1. Differential energy spectra of cosmic rays in the Earth's vicinity: average SPE spectrum for the last decades (SPE average) and for the years 1956 and 1989, and GCR spectra for solar minimum (e.g., 1965) and maximum (e.g., 1960) conditions.

the atmosphere for a longer time, resulting in a wider latitudinal distribution. Since the ${ }^{10} \mathrm{Be}$ abundance in polar ice represents both tropospheric and stratospheric production, a partial atmospheric mixing is usually considered, whose exact level is not known.

[7] Hence, we consider here two possible scenarios of the ${ }^{10} \mathrm{Be}$ production. One scenario, called the polar production henceforth, assumes that ${ }^{10} \mathrm{Be}$ measured in polar ice reflects only the polar production (above $60^{\circ}$ latitude). Another scenario, called mixing production, assumes that GCRrelated ${ }^{10} \mathrm{Be}$ consists of $50 \%$ stratospheric (i.e., globally mixed before deposition) and 50\% tropospheric (locally deposited) production [Masarik and Beer, 1999]. The SPE-related ${ }^{10} \mathrm{Be}$ is assumed to be produced only in the stratosphere, because of the lower energy of solar energetic particles, and is globally mixed. While the polar production yields the highest possible SPE signal, the mixing production corresponds to the lowest possible SPE signal, thus they both bound the realistic case. The ${ }^{14} \mathrm{C}$ production is global. Some results are shown in Table 1. Note that our computations of the ${ }^{14} \mathrm{C}$ and ${ }^{10} \mathrm{Be}$ production rate by GCR agree with earlier results [e.g., Masarik and Beer, 1999].

[8] While production of ${ }^{14} \mathrm{C}$ by GCR is mostly defined by capture of thermal neutrons by ${ }^{14} \mathrm{~N},{ }^{10} \mathrm{Be}$ is produced by energetic nucleons interacting with $\mathrm{N}$ and $\mathrm{O}$, leading to a lower production rate of the latter (see Table 1). The total production is dominated by GCR for both isotopes. Less energetic SPE-related particles cannot induce a fully developed cascade in the atmosphere and thus produce fewer neutrons leading to the smaller production of ${ }^{14} \mathrm{C}$. For ${ }^{10} \mathrm{Be}$ production, direct reactions by primaries are more important [see, e.g., Masarik and Reedy, 1995]. Let us consider the ratio of SPE- and GCR-related isotope production rates, $Q_{\mathrm{SPE}} / Q_{\mathrm{GCR}}$. Even though SPE-particles produce more ${ }^{14} \mathrm{C}$ then ${ }^{10} \mathrm{Be}$ nuclei, the relative contribution of SPE is larger for ${ }^{10} \mathrm{Be}\left(Q_{\mathrm{SPE}} / Q_{\mathrm{GCR}} \approx 1 \%\right.$ on average $)$ than for ${ }^{14} \mathrm{C}\left(Q_{\mathrm{SPE}} / Q_{\mathrm{GCR}} \approx 0.2 \%\right.$ - see Table 1$)$. Very strong SPEs

Table 1. Annual Atmospheric Production $Q$ (atoms $\mathrm{cm}^{-2} \mathrm{sec}^{-1}$ ) of Cosmogenic Isotopes Due to GCR for Solar Maximum and Minimum Conditions as Well as by the SPE Flux Averaged Over the Last Decades and for the Years 1956 and 1989 (see Figure 1)

\begin{tabular}{cccccc}
\hline Model & GCR Max & GCR Min & SPE Average & SPE 1956 & SPE 1989 \\
\hline${ }^{10} \mathrm{Be}$, polar & 0.022 & 0.051 & 0.002 & 0.021 & 0.017 \\
${ }^{10} \mathrm{Be}$, mix & 0.018 & 0.036 & 0.0002 & 0.003 & 0.002 \\
${ }^{14} \mathrm{C}$ & 1.5 & 2.5 & 0.005 & 0.08 & 0.05 \\
\hline
\end{tabular}

may contribute notably in the isotope annual production: from about $12 \%$ (mixing model) to $100 \%$ (polar model) in ${ }^{10} \mathrm{Be}$ and about $4 \%$ in ${ }^{14} \mathrm{C}$. Accordingly, ${ }^{10} \mathrm{Be}$ appears relatively more sensitive to SPEs than ${ }^{14} \mathrm{C}$. While changes of $F_{30}$ result in concurrent scaling of the SPE-related production rates of both ${ }^{10} \mathrm{Be}$ and ${ }^{14} \mathrm{C}$, any change of $P_{0}$ affects the two isotopes differently. Only SPEs with a hard spectrum can possibly affect ${ }^{14} \mathrm{C}$ production while ${ }^{10} \mathrm{Be}$ is also affected by softer events. This particularly concerns the polar production of ${ }^{10} \mathrm{Be}$.

\section{Results and Discussion}

\subsection{Recent Cycles}

[9] Let us evaluate, based on direct measurements of GCR and SPE during the last decades, the expected isotope production. The time profile of the calculated polar ${ }^{10} \mathrm{Be}$ production rate is shown in Figure 2a. One can see a significant isotope production by SPE during five periods $(1956,1960,1972,1989,2000)$ which are known to be very active. The annual $Q_{\mathrm{SPE}} / Q_{\mathrm{GCR}}$ ratio (the relative SPE effect) is shown in Figure $2 \mathrm{~b}$ for all models. The same five periods are prominent in all production rate series. However, from nearly doubling the polar production rate of ${ }^{10} \mathrm{Be}$, the SPE contribution decreases to $10-12 \%$ for the mixing ${ }^{10} \mathrm{Be}$ production and to a few $\%$ for ${ }^{14} \mathrm{C}$ (see Table 1). Unfortunately, a direct comparison with the measured isotope abundance is hardly possible for this period. ${ }^{14} \mathrm{C}$ data are distorted by nuclear tests after the 1950's and by the Suess effect since $1900 .{ }^{10} \mathrm{Be}$ data for the most recent times is quite rare because the top part of an ice core is usually missing for technical reasons.

\subsection{Last Centuries}

[10] The ${ }^{10} \mathrm{Be}$ data measured in the Dye-3 ice core (Greenland) [Beer et al., 1990] are shown in Figure 3 together with the expected production due to GCR [Usoskin et al., 2002] and the calculated SPE-related production (the mixing model). To compute SPE signal, we used dates and fluences of major SEP events reconstructed for the last centuries by McCracken et al. [2001a] using data on nitrates in polar ice. The computed annual production has been spread over three years with the proportion 1:2:1 to account for the stratospheric residence time of ${ }^{10} \mathrm{Be}[\mathrm{Beer}, 2000]$.

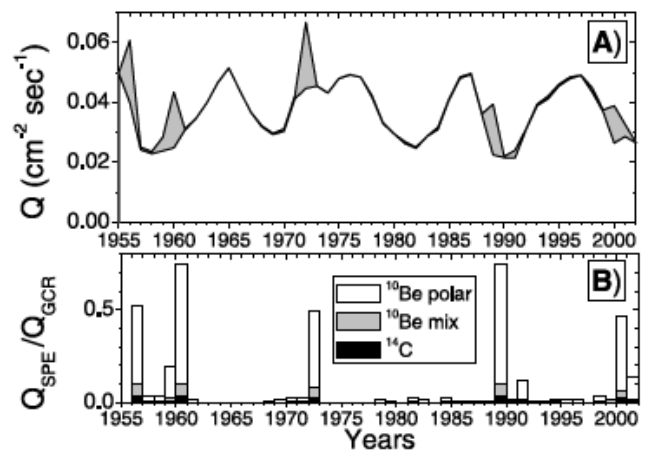

Figure 2. (a) Calculated polar production $Q$ of ${ }^{10} \mathrm{Be}$ by GCR (lower curve). Grey shading depicts additional ${ }^{10} \mathrm{Be}$ produced by SPE (see text). (b) Time profile of the ratio $Q_{\mathrm{SPE}} / Q_{\mathrm{GCR}}$ for the ${ }^{10} \mathrm{Be}$ polar, ${ }^{10} \mathrm{Be}$ mixing, and global ${ }^{14} \mathrm{C}$ production models (see the legend). 


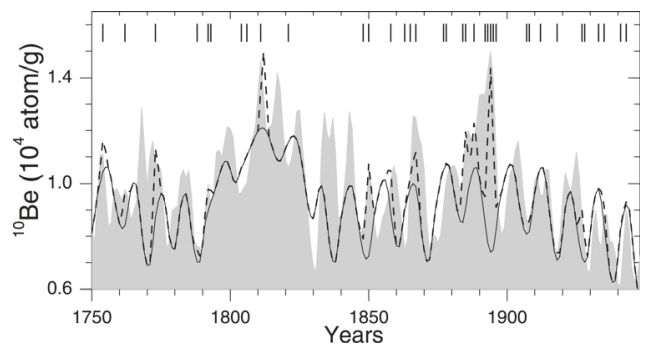

Figure 3. Time profile of the ${ }^{10} \mathrm{Be}$ concentration in polar ice. The measured concentration (Dye-3, Greenland [Beer et $a l ., 1990])$ is shown as grey shading. Solid and dashed lines represent the calculated ${ }^{10} \mathrm{Be}$ (mixing production) due to only GCR and GCR + SPE, respectively (see text). Bars on the top denote years with enhanced SPE fluence according to McCracken et al. [2001a].

Since the values of $P_{0}$ are not known, we used it as a free parameter to adjust the modeled ${ }^{10} \mathrm{Be}$ concentration to the measured one. Ten periods can be identified when peaks in ${ }^{10} \mathrm{Be}$ correspond to solar proton events: ca. 1755,1763 , 1774, 1793, 1813, 1851, 1859 (Carrington's flare), 1867, 1885-1896 (mostly 1895), 1927. Note that a possible contribution of large SPEs to ${ }^{10} \mathrm{Be}$ ca. 1895 was suggested earlier [McCracken et al., 2001b; Dorman, 2004].

[11] While only the mixing production model is shown, the polar production model yields similar results but allows a softer SEP spectrum. In order to reproduce the peaks in ${ }^{10} \mathrm{Be}$ (Figure 3), we used values of $P_{0}$ between 60 and $400 \mathrm{MV}$, i.e., similar to the recent decades. Including the SPE effect improves the correlation between calculated and measured ${ }^{10} \mathrm{Be}$ series, from 0.46 (confidence level 99\%) to $0.63(>99.99 \%)$. This analysis does not claim to reconstruct the parameters of SPEs in the past, but is only an illustration to show that some measured ${ }^{10} \mathrm{Be}$ peaks can be associated with SPEs. There is no one-to-one relationship, as only a dozen of 36 SPEs for 1750-1950 [McCracken et al., 2001a] can be related to ${ }^{10} \mathrm{Be}$ peaks, implying that not every SPE leads to enhanced isotope production. This can be due, e.g., to accompanying Forbush decreases (see Section 3.3). Also, some peaks, e.g., around 1768, 1817, $1834-1837$, in measured ${ }^{10} \mathrm{Be}$ are not accompanied by SEP events. This can be related to ${ }^{10} \mathrm{Be}$ deposition peculiarities, climatic effects or measurement errors in nitrates or ${ }^{10} \mathrm{Be}$. Moreover, the nitrate record is only an indirect proxy of strong SPEs whose efficiency is not $100 \%$ [Palmer et al., 2001].

[12] Major SPEs can produce peaks in ${ }^{10} \mathrm{Be}$ production during and around years of strong solar activity, when the GCR-related production is suppressed. This can conceivably lead to a 5.5 -year quasi-periodicity in ${ }^{10} \mathrm{Be}$ data (see Figure 2a between 1955 and 1965). A similar pattern was found recently in the actual ${ }^{10} \mathrm{Be}$ data $[$ McCracken et al., 2002]: An intermittent 5.5-year periodicity was apparent during some solar cycles and absent in others. McCracken et al. [2002] attributed this periodicity to an unspecified drift-related process of GCR modulation in the heliosphere. However, such a pattern is consistent with the SPE effect evaluated here (Figure 3): High (comparable with the 11-year modulation cycle magnitude) and short peaks in
${ }^{10} \mathrm{Be}$ production rate appearing around the maximum phase of some solar cycles. Therefore, the intermittent 5.5-year periodicity in ${ }^{10} \mathrm{Be}$ data can be naturally quantitatively explained by SPEs without a need for a further modulation process.

[13] A much lower SPE signal is expected in ${ }^{14} \mathrm{C}$, which would not exceed $10 \%$ of the GCR level even for the strongest event, while the amplitude of the 11-year cycle in ${ }^{14} \mathrm{C}$ production rate is about $35 \%$. Moreover, the complicated carbon cycle greatly attenuates high frequencies. For example, a year long $10 \%$ peak in the ${ }^{14} \mathrm{C}$ production rate would yield about 0.2 per mille enhancement in $\Delta^{14} \mathrm{C}$ which is below both the measurement uncertainty of about 2 per mille and the 11-year cycle of about 3 per mille. Assuming a severe SPE with a 2 per mille peak in $\Delta^{14} \mathrm{C}$ we would expect a doubling of the ${ }^{14} \mathrm{C}$ production rate and, correspondingly, a $4-10$-fold increase in ${ }^{10} \mathrm{Be}$ production depending on the used model. No such increase is observed in annual ${ }^{10} \mathrm{Be}$ during the last 500 years (Dye-3 series). Therefore, it is unlikely that such an enormous SPE took place in this period of time.

\subsection{Other Effects}

[14] In the above sections, all other conditions except for the changing particle flux during an SPE are assumed constant. However, this is not exactly true, since other phenomena can affect the isotope production by SPEs.

\subsubsection{Forbush Decreases}

[15] A strong SPE is often accompanied by a Forbush decrease (suppression of GCR flux by $10-30 \%$ during days-weeks) due to interplanetary transient phenomena, which may over-compensate an increase in isotope production due to the SPE. The relation between SPEs and Forbush decreases depends on the mutual Sun-Earth geometry and must be studied individually for each event. Accordingly, the present analysis can only provide upper limits for the SPE induced isotope production.

\subsubsection{Climatic Effects}

[16] Another factor which may introduce a spurious signal into the ${ }^{10} \mathrm{Be}$ record is the local climate, viz. the snow precipitation rate [e.g., Lal, 1987]. However, this factor, which may be responsible for some spikes or dips in the observed ${ }^{10} \mathrm{Be}$ data, is beyond the scope of this paper. 3.3.3. Geomagnetic Effects

[17] Interplanetary shocks, fast solar wind streams and enhanced interplanetary magnetic field, which often accompany strong SPEs, interact with the Earth's magnetosphere, resulting in a momentary decrease of the geomagnetic rigidity cutoff $P_{c}$ and enhancing the CR flux. These changes, however, are short and small [see, e.g., Kudo et al., 1987; Miyasaka et al., 2003], which makes them negligible on the annual scale. Long-term geomagnetic changes [e.g., Kudela and Bobik, 2004] are explicitly taken into account by the model.

\subsection{Solar Activity Reconstruction}

[18] Although the overall SPE production of the isotopes is negligible, ascribing a SPE-produced peak to GCR modulation may lead to an underestimate of solar activity for a particular period. A possible systematic offset can be avoided by setting the unknown proportionality between measured ${ }^{10} \mathrm{Be}$ concentration and ${ }^{10} \mathrm{Be}$ production rate. 
Thus, SPE-related effects only increase uncertainties of some individual points in the high-resolution reconstruction. These uncertainties can be considered as random because the SPE effect cannot be properly accounted and compensated in the past. In order to evaluate this, we have computed, using the method of Usoskin et al. [2003, 2004], sunspot number $N$, from the synthetic ${ }^{10} \mathrm{Be}$ series shown in Figure 3. The difference between the GCR and GCR+SPE models is $\sigma_{N}=4.2$, which is less than half of the uncertainties of the method. However, during solar cycle No. 13, when the strongest SPE effect is expected, the difference reaches up to 15 in sunspot number.

\section{Conclusions}

[19] Although the average contribution of solar proton events to the ${ }^{10} \mathrm{Be}$ production rate is negligible $(1-2 \%$ on average), extreme events may lead to detectable peaks in the annual ${ }^{10} \mathrm{Be}$ data. Ten episodes in ${ }^{10} \mathrm{Be}$ data can be identified during 1750-1950, which may hold signatures of strong SPEs. A combination of GCR and SPE signals may be responsible for a 5.5 -year periodicity in the ${ }^{10} \mathrm{Be}$ series found during some periods. A thorough study of the ${ }^{10} \mathrm{Be}$ data combined with the data on nitrates in polar ice may allow very strong SPEs to be identified. The possible effect of SPE in ${ }^{10} \mathrm{Be}$ production does not distort the long-term solar activity reconstructions based on the isotope data. Extreme events can, however, be observed on an inter-annual scale, and this provides a new possibility to study strong solar particle events in the past.

[20] The average production of ${ }^{14} \mathrm{C}$ by SPEs is negligible (less than $1 \%$ ), although extreme events may be marginally detectable in the high-resolution ${ }^{14} \mathrm{C}$ data. Accordingly, the SPE signal is not expected to distort the ${ }^{14} \mathrm{C}$-based reconstructions of solar activity.

[21] Acknowledgments. We thank Peter Foukal and Paul Damon for stimulating discussions, and Karel Kudela for consultations regarding the geomagnetic cutoff variations. Support from the Academy of Finland, the Finnish Academy of Science and Letters' Vilho, Yrjö and Kalle Väisälä Foundation, and the Russian Academy of Sciences are gratefully acknowledged.

\section{References}

Bartolomei, P., et al. (1995), Solar flare particle effect and seasonal radiocarbon variations in tree rings of the Northern and Southern hemispheres, Radiocarbon, 37, 593598.

Beer, J. (2000), Neutron monitor records in broader historical context, Space Sci. Rev., 93, 107119.

Beer, J., et al. (1990), Use of ${ }^{10} \mathrm{Be}$ in polar ice to trace the 11-year cycle of solar activity, Nature, 347, 164166.

Castagnoli, G., and D. Lal (1980), Solar modulation effects in terrestrial production of carbon-14, Radiocarbon, 22(2), 133158.

Damon, P. E., S. Cheng, and T. W. Linick (1989), Fine and hyperfine structure in the spectrum of secular variations of atmospheric ${ }^{14} \mathrm{C}$, Radiocarbon, 31, 704718 .

Dorman, L. I. (2004), Cosmic Rays in the Earth's Atmosphere and Underground, 855 pp., Springer, New York.

Feynman, J., G. Spitale, J. Wang, and S. Gabriel (1993), Interplanetary proton fluence model: JPL 1991, J. Geophys. Res., 98(A8), 13,281 13,294.

Freier, P. S., and W. R. Webber (1963), Exponential rigidity spectrums for solar-flare cosmic rays, J. Geophys. Res., 68, 16051629.
Jöckel, P., C. A. M. Brenninkmeijer, M. G. Lawrence, and P. Siegmund (2003), The detection of solar proton produced ${ }^{14} \mathrm{CO}$, Atmos. Chem. Phys., 3, 17331752.

Kudela, K., and P. Bobik (2004), Long-term variations of geomagnetic rigidity cutoffs, Solar Phys., 224, 423431.

Kudo, S., M. Wada, P. Tanskanen, and M. Kodama (1987), Local time and cutoff rigidity dependence of storm time increase associated with geomagnetic storms, J. Geophys. Res., 92, 47194724.

Lal, D. (1987), ${ }^{10} \mathrm{Be}$ in polar ice: Data reflect changes in cosmic ray flux or polar meteorology, Geophys. Res. Lett., 14, 785788.

Lingenfelter, R. E., and R. Ramaty (1970), Astrophysical and geophysical variations in C-14 production, in Proceedings of the 12th Nobel Symposium, Radiocarbon Variations and Absolute Chronology, edited by I. U. Olsson, pp. 513 537, John Wiley, Hoboken, N. J.

Masarik, J., and J. Beer (1999), Simulation of particle fluxes and cosmogenic nuclide production in the Earth's atmosphere, J. Geophys. Res., 104(D10), 12,099 12,111.

Masarik, J., and R. C. Reedy (1995), Terrestrial cosmogenic-nuclide production systematics calculated from numerical simulations, Earth Planet. Sci. Lett., 136, 381395.

McCracken, K. G., et al. (2001a), Solar cosmic ray events for the period 1561 1994: 1. Identification in polar ice, 1561 1950, J. Geophys. Res., 106(A10), 21,585 21,598.

McCracken, K. G., D. F. Smart, M. A. Shea, and G. A. M. Dreschhoff (2001b), 400 years of large fluence solar proton events, Conf. Pap. Int. Cosmic Ray Conf., 8, 32093212.

McCracken, K. G., J. Beer, and F. B. McDonald (2002), A five-year variability in the modulation of the galactic cosmic radiation over epochs of low solar activity, Geophys. Res. Lett., 29(24), 2161, doi:10.1029/ 2002 GL015786.

McCracken, K. G., F. B. McDonald, J. Beer, G. Raisbeck, and F. Yiou (2004), A phenomenological study of the long-term cosmic ray modulation, 8501958 AD, J. Geophys. Res., 109, A12103, doi:10.1029/ 2004JA010685.

Miyasaka, H., et al. (2003), Geomagnetic cutoff variation observed with TIBET neutron monitor, Conf. Pap. Int. Cosmic Ray Conf., 28, 3609 3612 .

Palmer, A. S., T. D. van Ommen, M. A. J. Curran, and V. Morgan (2001), Ice-core evidence for a small solar-source of atmospheric nitrate, Geophys. Res. Lett., 28(10), 19531956.

Shea, M. A., and D. F. Smart (1990), A summary of major solar proton events, Sol. Phys., 127, 297320.

Solanki, S. K., et al. (2004), An unusually active Sun during recent decades compared to the previous 11,000 years, Nature, 431, 10841087.

Stuiver, M., and T. F. Braziunas (1998), Antropogenic and solar components of hemispheric ${ }^{14}$ C, Geophys. Res. Lett., 25, 329332.

Usoskin, I. G., et al. (2002), Physical reconstruction of cosmic ray intensity since 1610, J. Geophys. Res., 107(A11), 1374, doi:10.1029/ 2002JA009343.

Usoskin, I. G., et al. (2003), Millennium-scale sunspot number reconstruction: Evidence for an unusually active Sun since the 1940s, Phys. Rev. Lett., 91(21), 211101.

Usoskin, I. G., et al. (2004), Reconstruction of solar activity for the last millennium using ${ }^{10} \mathrm{Be}$ data, Astron. Astrophys., 413, 745751.

Usoskin, I. G., K. Alanko-Huotari, G. A. Kovaltsov, and K. Mursula (2005), Heliospheric modulation of cosmic rays: Monthly reconstruction for 1951 2004, J. Geophys. Res., 110, A12108, doi:10.1029/ 2005JA011250.

Webber, W. R., and P. R. Higbie (2003), Production of cosmogenic Be nuclei in the Earth's atmosphere by cosmic rays: Its dependence on solar modulation and the interstellar cosmic ray spectrum, J. Geophys. Res., 108(A9), 1355, doi:10.1029/2003JA009863.

J. Beer, Department of Surface Water, EAWAG, CH-8600 Dübendorf, Switzerland.

G. A. Kovaltsov, Ioffe Physical-Technical Institute, Politekhnicheskaya 26, RU-194021 St. Petersburg, Russia.

B. Kromer, Heidelberg Akademie der Wissenschaften, D-69120 Heidelberg, Germany.

S. K. Solanki, Max-Planck-Institut für Sonnensystemforschung, D-37191 Katlenburg-Lindau, Germany.

I. G. Usoskin, Sodankylä Geophysical Observatory (Oulu Unit), P.O. Box 3000, University of Oulu, FIN-90014 Oulu, Finland. (ilya. usoskin@oulu.fi) 